\title{
Deviations from tri-bimaximal mixing
}

\author{
W. Rodejohann ${ }^{\mathrm{a} *}$ \\ ${ }^{a}$ Max-Planck-Institut für Kernphysik, Postfach 103980, 69029 Heidelberg, Germany
}

Current data indicates that lepton mixing is very close to the tri-bimaximal mixing scenario. In general, however, one expects deviations from any mixing scenario. We discuss several examples to perturb tri-bimaximal mixing, namely soft breaking, renormalization group running and charged lepton corrections. We also present a convenient parametrization of the PMNS matrix which takes advantage of the apparently close to tri-bimaximal mixing pattern. Finally, it is discussed how to generate values of $U_{e 3}$ which correspond to the recently found hint for non-zero $U_{e 3}$.

\section{Introduction}

Neutrino physics has entered the precision era. In particular, the entries of the unitary PMNS matrix $U$ are known with quite good precision. In general,

$U=R_{23}\left(\theta_{23}\right) \tilde{R}_{13}\left(\theta_{13} ; \delta\right) R_{12}\left(\theta_{12}\right) P$,

where $R_{i j}(\theta)$ is a rotation with angle $\theta$ around the $i j$-axis, e.g.,

$R_{12}\left(\theta_{12}\right)=\left(\begin{array}{ccc}c_{12} & s_{12} & 0 \\ -s_{12} & c_{12} & 0 \\ 0 & 0 & 1\end{array}\right)$

and

$\tilde{R}_{13}\left(\theta_{13} ; \delta\right)=\left(\begin{array}{ccc}c_{13} & 0 & s_{13} e^{-i \delta} \\ 0 & 1 & 0 \\ -s_{13} e^{i \delta} & 0 & c_{13}\end{array}\right)$.

Here $c_{i j}=\cos \theta_{i j}, \quad s_{i j}=\sin \theta_{i j} \quad$ with $\delta$ the CP-violating Dirac phase and $P=$ $\operatorname{diag}\left(1, e^{-i \alpha_{2} / 2}, e^{-i \alpha_{3} / 2}\right)$ contains the Majorana phases. Harrison, Perkins, and Scott first emphasized that the experimentally obtained mixing matrix is close to the simple tri-bimaximal mixing (TBM) form [1:

$$
\begin{aligned}
& U_{\mathrm{TBM}}=R_{23}(\pi / 4) \\
& =\left(\begin{array}{ccc}
\sqrt{\frac{2}{3}} & \sqrt{\frac{1}{3}} & 0 \\
-\sqrt{\frac{1}{6}} & \sqrt{\frac{1}{3}} & \sqrt{\frac{1}{2}} \\
\sqrt{\frac{1}{6}} & -\sqrt{\frac{1}{3}} & \sqrt{\frac{1}{2}}
\end{array}\right),
\end{aligned}
$$

*Work supported by the ERC under the Starting Grant MANITOP and the DFG in the Transregio 27. where $\theta_{\mathrm{TBM}}=\sin ^{-1} \sqrt{\frac{1}{3}}$. The apparent closeness to the TBM scenario invites to propose a parametrization of the PMNS matrix with TBM as the zeroth order expression. This "triminimal" parametrization is 2] (see also [3]):

$U=R_{23}(\pi / 4) R_{23}\left(\epsilon_{23}\right) \tilde{R}_{13}\left(\epsilon_{13} ; \delta\right) R_{12}\left(\epsilon_{12}\right) R_{12}\left(\theta_{\mathrm{TBM}}\right)$,

with small $\epsilon_{i j}$. The virtues of triminimality are (i) each small $\epsilon_{i j}$ is responsible for the deviation of, and only of, $\theta_{i j}$ from its tri-bimaximal value; (ii) the element $U_{e 3}$ is $\sin \epsilon_{13} e^{-i \delta}$ and therefore possesses exactly the form as promulgated in the PDG-description of mixing matrices; (iii) is it obviously unitary.

The other mixing observables besides $U_{e 3}$ are

$$
\begin{aligned}
\sin ^{2} \theta_{12}=\left(\cos \epsilon_{12}+\sqrt{2} \sin \epsilon_{12}\right)^{2} & \simeq \frac{1}{3}+\frac{2 \sqrt{2}}{3} \epsilon_{12}, \\
\sin ^{2} \theta_{23}=\frac{1}{2}+\sin \epsilon_{23} \cos \epsilon_{23} & \simeq \frac{1}{2}+\epsilon_{23} .
\end{aligned}
$$

\section{The mass matrix and deviations from TBM}

The Majorana mass matrix is defined as $m_{\nu}=$ $U^{*} \operatorname{diag}\left(m_{1}, m_{2}, m_{3}\right) U^{\dagger}$ and takes a quite simple form in case of TBM:

$\left(m_{\nu}\right)^{\mathrm{TBM}}=\left(\begin{array}{ccc}A & B & B \\ \cdot & \frac{1}{2}(A+B+D) & \frac{1}{2}(A+B-D) \\ \cdot & \cdot & \frac{1}{2}(A+B+D)\end{array}\right)$,

where $A, B, D$ are functions of the masses and Majorana phases, see, e.g., [5]. The given matrix is in fact obtained from a slightly modified (but physically identical) $U_{\mathrm{TBM}}$, which corresponds to choosing $\theta_{23}=-\pi / 4$ in the standard 


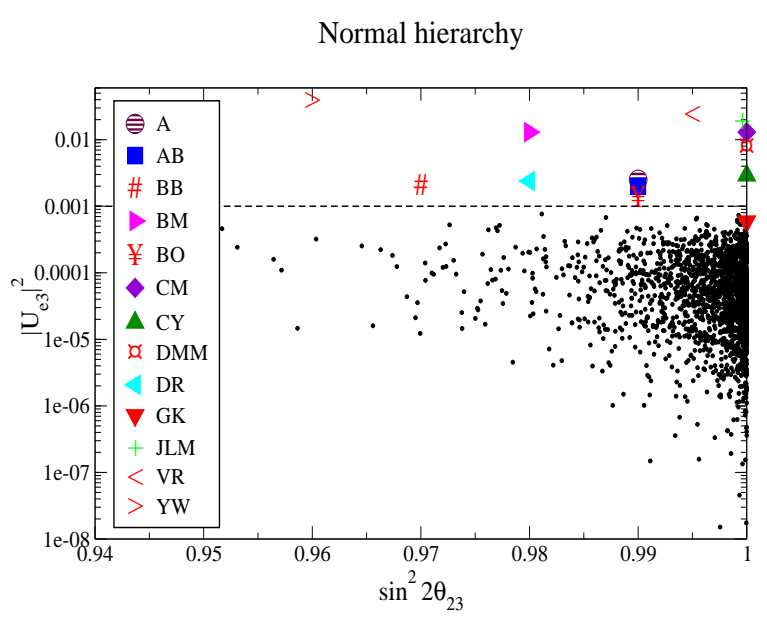

Figure 1. Scatter plot of $\left|U_{e 3}\right|^{2}$ against $\sin ^{2} 2 \theta_{23}$ from a perturbed TBM mass matrix. Shown also are the results of successful $S O(10)$ GUTs. Taken from [5].

PDG-description of eq. (11). The most straightforward way to perturb TBM is to perturb every mass matrix entry, i.e., $\left(m_{\nu}\right)_{e e}=A\left(1+\epsilon_{e e}\right)$, $\left(m_{\nu}\right)_{e \mu}=B\left(1+\epsilon_{e \mu}\right)$ and so on. In total, there are six independent $\epsilon_{\alpha \beta}$, all of which are complex: $\epsilon_{\alpha \beta}=\left|\epsilon_{\alpha \beta}\right| e^{i \phi_{\alpha \beta}}$. We bound in the analysis, described in detail in $\left[5,\left|\epsilon_{\alpha \beta}\right| \leq 0.2\right.$ and vary the phases between zero and $2 \pi$. The implied deviations from TBM depend strongly on the neutrino mass ordering and scale. We find that the magnitude of $\theta_{13}$ is the most sensitive parameter. Atmospheric neutrino mixing is less sensitive, while $\theta_{12}$ has nothing to say at all. For a normal hierarchy of neutrino masses, $\left|U_{e 3}\right|^{2}$ has a maximal value of $\frac{4}{9} R|\epsilon|^{2} \simeq 7 \cdot 10^{-4}$, where $\epsilon$ is one of the $\epsilon_{\alpha \beta}$ and $R$ is the ratio of solar and atmospheric mass-squared differences. Fig. 11 shows a scatter plot resulting from the analysis. It compares broken TBM also with the predictions from 13 successful $S O(10)$ GUTs, almost all of which predict $\left|U_{e 3}\right|^{2} \geq 10^{-3}$. In the inverted hierarchy, in turn, broken TBM can lead to $\left|U_{e 3}\right|^{2}$ proportional to $|\epsilon|^{2}$ without suppression through $R$. It can therefore be as large as 0.01 , while for quasi-degenerate neutrinos the complete allowed parameter space can be covered. All these features allow to distinguish GUTs from broken TBM if the mass ordering is known. The last two cases have an interesting correlation with neutrino-less double beta decay: if there is no cancellation in the effective mass $\left|\left(m_{\nu}\right)_{e e}\right|$, then $\theta_{13}$ is negligible, while for maximal cancellation $\theta_{13}$ can reach or exceed 0.1. With "no cancellation" we denote the situation in which $\left|\left(m_{\nu}\right)_{e e}\right| / m_{\max } \simeq 1$, while "maximal cancellations" have occurred when $\left|\left(m_{\nu}\right)_{e e}\right| / m_{\max } \simeq$ $\cos 2 \theta_{12}$, where $m_{\max }$ is the largest neutrino mass.

In principle included in the presented analysis are radiative corrections, because they can be estimated with good precision by zero $\epsilon_{e e, e \mu, \mu \mu}$ and $\epsilon_{e \tau}=\epsilon_{\mu \tau}=\frac{1}{2} \epsilon_{\tau \tau} \equiv \epsilon_{\mathrm{RG}}=c \frac{m_{\tau}^{2}}{16 \pi v^{2}} \ln M_{X} / m_{Z}$ with $c=-\frac{3}{2}$ and $c=1+\tan ^{2} \beta$ in the SM and MSSM, respectively. In Ref. [4 it was shown that RG running leads to $\theta_{i j}=\theta_{i j}^{\mathrm{TBM}}+k_{i j} \epsilon_{\mathrm{RG}}$. Here $\theta_{i j}^{\mathrm{TBM}}$ are the mixing angles in case of TBM, and

$$
\begin{gathered}
k_{12}=\frac{\sqrt{2}}{3} \frac{\left|m_{1}+m_{2} e^{i \alpha_{2}}\right|^{2}}{m_{2}^{2}-m_{1}^{2}}, \\
k_{23}=-\left(\frac{2}{3} \frac{\left|m_{2}+m_{3} e^{i\left(\alpha_{3}-\alpha_{2}\right)}\right|^{2}}{m_{3}^{2}-m_{2}^{2}}+\frac{1}{3} \frac{\left|m_{1}+m_{3} e^{i \alpha_{3}}\right|^{2}}{m_{3}^{2}-m_{1}^{2}}\right), \\
k_{13}=-\frac{\sqrt{2}}{3}\left(\frac{\left|m_{2}+m_{3} e^{i\left(\alpha_{3}-\alpha_{2}\right)}\right|^{2}}{m_{3}^{2}-m_{2}^{2}}-\frac{\left|m_{1}+m_{3} e^{i \alpha_{3}}\right|^{2}}{m_{3}^{2}-m_{1}^{2}}\right) .
\end{gathered}
$$

The sign and the size of the RG effects can be easily estimated now, depending of course on the neutrino mass ordering and hierarchy. To give one example, a value of $\left|U_{e 3}\right| \simeq 0.1$ requires typically neutrino masses $\gtrsim 0.1 \mathrm{eV}$ and $\tan \beta \gtrsim 15$. In this case one requires a suppression of $k_{12}$ through $\alpha_{2}$ to keep $\theta_{12}$ under control. This is equivalent to maximal cancellation in the effective mass governing neutrino-less double beta decay.

\section{Deviating TBM with charged leptons}

Charged lepton corrections are quite popular when it comes to perturbing mixing scenarios 6 , 7889. . Using the relation $U=U_{\ell}^{\dagger} U_{\nu}$, it is usually assumed that $U_{\nu}$ corresponds to TBM and $U_{\ell}$ is, GUT-inspired, CKM-like. This means that it is given only by a 12 -rotation with angle $\sin ^{-1} \lambda$. In this case, one can show that

$$
\begin{gathered}
\sin ^{2} \theta_{12} \simeq \frac{1}{3}-\frac{2}{3} \lambda \cos \phi \\
\left|U_{e 3}\right| \simeq \frac{1}{\sqrt{2}} \lambda, \quad J_{\mathrm{CP}}=\frac{\lambda}{6} \sin \phi, \\
\sin ^{2} \theta_{23} \simeq \frac{1}{2}-\mathcal{O}\left(\lambda^{2}\right) .
\end{gathered}
$$


Here $\phi$ is a phase showing up in $U_{\nu}$ and $J_{\mathrm{CP}}$ is the Jarlskog invariant for neutrino oscillations. Hence, there is a direct correlation between $U_{e 3}$, $\sin ^{2} \theta_{12}$ and $\mathrm{CP}$ violation. In particular, if $\lambda$ is large, e.g., the sine of the Cabibbo angle, then its contribution to $\sin ^{2} \theta_{12}$ is dangerously large unless we suppress it with $\phi=\pi / 2$. In this case, the CP phase is maximal [8].

There is an alternative way of charged lepton corrections, namely when $U_{\ell}^{\dagger}$ corresponds to TBM and $U_{\nu}$ is CKM-like [9]. In this case, there is a correlation in analogy to the one above, but now with atmospheric neutrino mixing, $\left|U_{e 3}\right|$ and $\mathrm{CP}$ violation. It is harder to test because $\left|U_{e 3}\right| \propto \lambda^{2}$ is smaller than in the previous case.

\section{A hint for non-zero $\theta_{13}$}

Recently a hint for non-zero $U_{e 3}$ emerged from global fits of neutrino data 1011]. Ref. [10] determines a $1 \sigma$ range of $\sin ^{2} \theta_{13}=0.016 \pm 0.010$, with a total significance of $1.6 \sigma$ for non-zero $\theta_{13}$. From the previous discussion 2 it is clear that values of $\left|U_{e 3}\right| \simeq 0.1$ can be obtained by:

- soft breaking of a TBM (or a $\mu-\tau$ symmetric) mass matrix in case of an inverted hierarchy or quasi-degenerate neutrinos. A characteristic signature in massrelated neutrino experiments, in particular neutrino-less double beta decay, follows in these regimes;

- charged lepton corrections to TBM from $U_{\ell}$ with $\lambda \simeq \sqrt{2}\left|U_{e 3}\right|$, a number interestingly not too far away from the sine of the Cabibbo angle (or one third of it). In fact, the $1 \sigma$ range given above corresponds to $\lambda=0.18_{-0.07}^{+0.05}$. A characteristic correlation with solar neutrino mixing and $\mathrm{CP}$ violation $(\delta \simeq \pi / 2$ or $3 \pi / 2)$ is obtained;

- RG corrections to TBM in the MSSM with sizable $m_{i} \gtrsim 0.1 \mathrm{eV}$ and $\tan \beta \gtrsim 15$. Cancellations in the effective mass are required in order to suppress running of $\theta_{12}$. Note that MSSM-related lepton flavor violating processes typically grow with $\tan \beta$.

\footnotetext{
${ }^{2}$ We should stress however that sizable values of $\theta_{13}$ are
} typical also for GUTs.
It will be interesting to see if the currently weak hint survives or becomes more significant. In the likely case of one of the deviation possibilities being dominant, future precision data will then help us distinguishing them. In any case, the value of $\left|U_{e 3}\right|$ used here can be regarded as an interesting and testable benchmark scenario.

I wish to thank my co-authors for fruitful collaborations and the organizers of NOW 2008, E. Fogli and G.L. Lisi, for their invitation and for hosting a stimulating workshop.

\section{REFERENCES}

1. P. F. Harrison, D. H. Perkins and W. G. Scott, Phys. Lett. B 530, 167 (2002); Phys. Lett. B 535, 163 (2002); Z. Z. Xing, Phys. Lett. B 533, 85 (2002); X. G. He and A. Zee, Phys. Lett. B 560, 87 (2003).

2. S. Pakvasa, W. Rodejohann and T. J. Weiler, Phys. Rev. Lett. 100, 111801 (2008).

3. S. F. King, Phys. Lett. B 659, 244 (2008).

4. A. Dighe, S. Goswami and P. Roy, Phys. Rev. D 76, 096005 (2007); A. Dighe, S. Goswami and W. Rodejohann, Phys. Rev. D 75, 073023 (2007).

5. C. H. Albright and W. Rodejohann, Phys. Lett. B 665, 378 (2008).

6. S. F. King, JHEP 0209, 011 (2002); C. Giunti and M. Tanimoto, Phys. Rev. D 66, 113006 (2002); S. Antusch and S. F. King, Phys. Lett. B 631, 42 (2005); S. Boudjemaa and S. F. King, arXiv:0808.2782 [hep-ph].

7. P. H. Frampton, S. T. Petcov and W. Rodejohann, Nucl. Phys. B 687, 31 (2004).

8. F. Plentinger and W. Rodejohann, Phys. Lett. B 625, 264 (2005).

9. K. A. Hochmuth, S. T. Petcov and W. Rodejohann, Phys. Lett. B 654, 177 (2007).

10. G. L. Fogli, E. Lisi, A. Marrone, A. Palazzo and A. M. Rotunno, Phys. Rev. Lett. 101, 141801 (2008).

11. A. B. Balantekin and D. Yilmaz, J. Phys. G 35, 075007 (2008); H. L. Ge, C. Giunti and Q. Y. Liu, arXiv:0810.5443 [hep-ph]. 